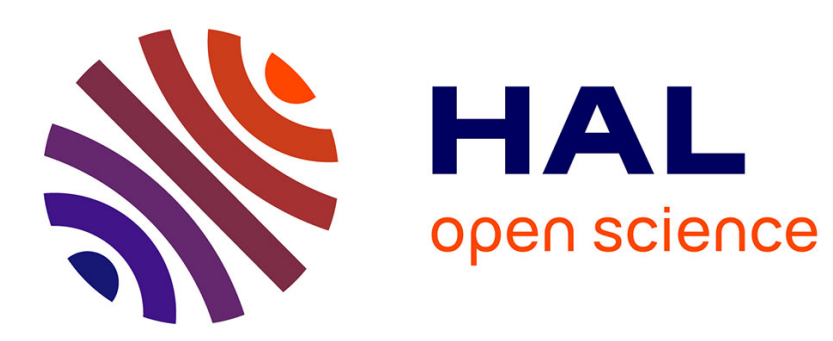

\title{
Jeux de différences. Une approche méthodologique de l'identité à l'épreuve de deux terrains varois
}

Annie-Hélène Dufour, Thomas K Schippers

\section{To cite this version:}

Annie-Hélène Dufour, Thomas K Schippers. Jeux de différences. Une approche méthodologique de l'identité à l'épreuve de deux terrains varois. Le monde alpin et rhodanien, 1993, 21 (1), pp.169 - 187. 10.3406/mar.1993.1508 . hal-01853259

\section{HAL Id: hal-01853259 \\ https://hal.science/hal-01853259}

Submitted on 2 Aug 2018

HAL is a multi-disciplinary open access archive for the deposit and dissemination of scientific research documents, whether they are published or not. The documents may come from teaching and research institutions in France or abroad, or from public or private research centers.
L'archive ouverte pluridisciplinaire $\mathbf{H A L}$, est destinée au dépôt et à la diffusion de documents scientifiques de niveau recherche, publiés ou non, émanant des établissements d'enseignement et de recherche français ou étrangers, des laboratoires publics ou privés. 


\section{Jeux de différences. Une approche méthodologique de l'identité à} l'épreuve de deux terrains varois

Annie-Hélène Dufour, Thomas K. Schippers

\section{Citer ce document / Cite this document :}

Dufour Annie-Hélène, Schippers Thomas K. Jeux de différences. Une approche méthodologique de l'identité à l'épreuve de deux terrains varois. In: Le Monde alpin et rhodanien. Revue régionale d'ethnologie, n¹-2/1993. L'identité vécue. Discours, rites, emblèmes (Provence, Languedoc, Hautes-Alpes) pp. 169-187;

doi : https://doi.org/10.3406/mar.1993.1508

https://www.persee.fr/doc/mar_0758-4431_1993_num_21_1_1508

Fichier pdf généré le 19/07/2018 


\begin{abstract}
Dufour (Annie-Hélène) et Schippers (Thomas K.). — Games of différences. A methodological approach assessed using two case studies of Var.

After briefly reviewing how ethnologists have used the concept of identity in France, the authors propose an original methodological approach to it. They then present how this two case studies method is applied in the Provence region (South-East France).

They set out to analyse the feelings of local belon-ging, refiising any a priori definition of identity and relying on three sets of eues : indicators, markers and stereotypes. These allow a distinction between the more objective marks of differentiation on the one hand and more the subjective criteria of selfidentification autochtony on the other. The feelings of belonging are not analysed as a static phenomenon but as a resuit of dynamic interactions between these sets of references. Applying these methodological tools to two contrasted case studies within the same region, shows the possible heuristic interest of this approach.
\end{abstract}

Le Monde Alpin et rhodanien, ler-2 trimestres 1993, L'identité vécue. Discours, rites, emblèmes, pp. 169 à 187.

\title{
Résumé
}

Dufour (Annie-Hélène) et Schippers (Thomas K.). - Jeux de différences. Une approche méthodologique de l'identité à l'épreuve de deux terrains varois.

Après avoir évoqué les grandes étapes historiques de l'utilisation du concept d'identité par les ethnologues du domaine français, les auteurs proposent une réflexion méthodologique autour de ce concept à travers deux études de cas situés en Provence intérieure.

En refusant une définition a priori de l'identité, les auteurs ont abordé les sentiments d'appartenance à l'aide de trois séries d'indices : indicateurs, marqueurs et stéréotypes. Ces indices leur ont permis de saisir à la fois des traits objectif de différenciation et des critères d'identification autochtones plus subjectifs. Ainsi les sentiments d'appartenance sont-ils analysés non pas comme un phénomène statique mais comme la résultante de relations dynamiques qu 'entretiennent entre eux des ensembles de traits. La mise en œuvre de ces outils dans deux situations contrastées au sein d'une même aire géographique illustre l'intérêt heuristique de l'approche.

Le Monde Alpin et rhodanien, ler-2 trimestres 1993, L'identité vécue. Discours, rites, emblèmes, pp. 169 à 187. 


\title{
Jeux de différences
}

\section{Une approche méthodologique de l'identité à l'épreuve de deux terrains varois*}

\author{
Annie-Hélène Dufour, Thomas K. Schippers
}

$\mathrm{L}^{\prime}$

histoire de la discipline ethnologique est jalonnée de carcasses rouillées, de notions et de concepts abandonnés après avoir servi aux chercheurs de véhicule à leur pensée pendant quelques années, voire quelques décennies. Mais cette histoire connait aussi ses bricoleurs qui s'en retournent chercher une épave trop vite délaissée pour lui donner une seconde jeunesse munie de nouveaux équipements. Structure, culture, ethnicité, identité figurent parmi ces concepts ou notions qui ont connu depuis une cinquantaine d'années des come-back périodiques. Ainsi, sous le concept d'identité, qui n’apparaît véritablement dans le vocabulaire ethnologique du domaine français qu’à la fin des années 1960 , se dissimulent des interrogations bien plus anciennes sur les spécificités de certains phénomènes ou groupes sociaux.

Comme l'a souligné Christian Bromberger (1993), nous retrouvons déjà dans certains projets de recherche lancés par les fondateurs du Musée National des Arts et Traditions Populaires un intérêt qu'on a pu qualifier de substantiviste pour les différences et les spécificités régionales : inventaires raisonnés des formes d'habitat rural, du mobilier domestique ou encore de techniques et outils agricoles. Ces inventaires de traits souvent fondés sur des faits relevant de la culture matérielle mais pas exclusivement, cherchaient à définir des typologies et à les transcrire dans l'espace géographique. L'abandon des recherches extensives au profit de monographics localcs après la deuxième guerre mondiale aboutira quelques vingt années plus tard à un regain d'intérêt pour la spécificité locale ou régionale, mais cette fois-ci étudiée à travers sa perception autochtone. C'est à partir de cette époque, où l'ethnologie de la France s'intéresse beaucoup au vécu et aux discours des populations, que les spécificités locales et régionales (perçues) seront affu-

* A l'origine de cer article se trouve une recherche menée en 1983-1984 avec le soutien financier de la Mission du Patrimoine Ethnologique (Ministère de la Culture) er de l'URA 1346 du CNRS, dans le cadre d'un appel d'offres intitulé "Identité culturelle, appartenance régionale ". 
blées du nom d'identité. La reprise de ce terme dans les contextes les plus variés de la société française des années 1970-1980 aura alors comme conséquence son abandon ou du moins une grande méfiance à son égard de la part de la majorité des ethnologues français.

C'est dans cette situation où le concept d'identité est à la fois considéré comme galvaudé et néanmoins utilisé par les ethnologues, ne serait-ce que pour satisfaire une demande sociale ${ }^{(1)}$, que nous avons entrepris un travail sur les identités micro-régionales de deux cantons varois, dans le but de mettre à l'épreuve de ces deux terrains une méthode d'approche de la notion d'identité fondée sur des séries d'indices préalablement définis. Il va sans dire que, dans une telle étude, le choix de l'unité administrative du canton ne pouvait être limitatif. Notion commode au départ, celle-ci a été élargie en fonction des observations en cours d'enquête. Les lieux sur lesquels a porté notre interrogation, contrairement à d'autres " pays " provençaux clairement délimités culturellement et spatialement comme le pays d'Arles, la Crau, la Camargue, le pays d'Apt, le pays d'Aigues, etc., appartiennent à ce Var intérieur qui a échappé à tout découpage. Identifier et délimiter des sentiments d'appartenance dans ces micro-régions sans nom particulier et qui ne se désignent pas d'elles-mêmes au regard de l'observateur fut par ailleurs un défi supplémentaire. Labsence dans cette région de revendications identitaires nous a semblé propice à une observation des mécanismes de définition de Soi et des Autres dans un contexte où l'identité n'est pas vécue comme un enjeu.

Pour appréhender cette notion dans toute sa complexité, on a procédé à l'analyse et la comparaison des trois séries d'indices que sont les indicateurs, les marqueurs, et les stéréotypes, selon une description esquissée par Christian Bromberger (1988), s'inspirant de l'analyse du sociolinguiste W. Labov.

- Les indicateurs sont des traits " objectifs " dont l'extension et la superposition dans l'espace permettent de délimiter des unités subculturelles; construits par l'observateur au cours d'une analyse substantiviste des faits, ils forment une série ouverte et ne sont pas nécessairement perçus comme marques de différence par les usagers. L'analyse contrastive de ces indicateurs a porté :

- sur les aires d'interrelations matrimoniales, économiques (marchés et foires, pôles de rencontre des habitants d'un même " pays "...), festives et religieuses (zone d'attraction des fêtes locales, des pèlerinages...), les aires d'interconnaissance, de maitrise toponymique, de sobriquets collectifs, d'intercompréhension dialectale, etc. ;

- sur les formes de production agropastorales, de consommation (habitation, cuisine...), de sociabilité, de coopération technique...

- sur les pratiques linguistiques (dialecte, français régional...) en complément des enquêtes phonétiques et lexicales entreprises dans le cadre de l'Atlas linguistique et ethnographique de la Provence (A.L.P.) ${ }^{(2)}$.

(1) Soutenue par des crédits de recherche.

(2) Les enquêtes linguistiques ont été faites en collaboration avec C. Martel, dialectologue au CNRS et coresponsable de l'A.L.P. 
- Les marqueurs sont des traits perçus, reconnus et retenus par les usagers comme différenciateurs et dont l'extension trace les limites de l'espace emblématique de l'entité d'appartenance. Contrairement aux indicateurs, les marqueurs forment une série fermée et hiérarchisée. Ils peuvent être des traits de la culture matérielle, se référer à des comportements collectifs, à des pratiques linguistiques, à des lieux ou à des personnages emblématiques... Certains marqueurs peuvent donc être des indicateurs reconnus tandis que d'autres semblent dépourvus de fondements " objectifs".

- Les stéréotypes sont des jugements de valeurs sur l'en-groupe et l'horsgroupe de référence, s'exprimant à travers des attitudes, des comportements collectifs (vêtements, cuisine, " morale "...), des sobriquets, le discours sur "l'étranger "...

"L'étude des indicateurs renvoie donc aux propriétés différentielles des groupes, celle des marqueurs et stéréotypes à la conscience d'appartenance " (Bromberger, 1985). Cette triple approche a permis de comparer le contenu et les frontières des identités locales et micro-régionales, telles qu'elles sont vécues et perçues et telles que les révèle l'analyse contrastive des indicateurs, ce que l'anthropologie culturelle américaine des années 1970 aurait qualifié de point de vue emic et de point de vue etic. Ainsi, le sentiment d'appartenance est analysé non pas comme un phénomène statique mais plutôt comme la résultante des relations dynamiques qu'entretiennent entre eux des ensembles de traits.

\section{L'IDENTITÉ À LA LOUPE}

Traits objectifs de différenciation, les indicateurs forment, comme nous venons de le souligner, une série non-limitée a priori. Leur choix et l'évaluation de la pertinence de ces choix sont le fait de l'observateur extérieur qui recherche des indices de différenciation interne et externe au groupe étudié. Un premier indicateur retenu fut celui de la situation géographique. Le canton de Cotignac, au centre du département du Var, est formé d'une série de communes, chacune composée d'un gros bourg central (3) entouré d'une zone de culture (champs et surtout vignes) et d'un espace boisé. Bien que de tailles différentes, les communes du canton partagent une certaine structure composée de cette trilogie d'espaces : un habitat groupé, des terres cultivées et des " collines "(4). Cette similarité de composition des espaces géographiques des communes du canton ne constitue pas pour autant un ensemble qui trancherait sur un extérieur très distinct. Au contraire, elle s'inscrit dans une vaste zone qui s'étend d'est en ouest depuis l'arrière-pays antibois jusqu'aux environs d'Aix-en-Provence et où l'on retrouve, pas seulement

(3) Il s'agit d'une forme d'habitat typiquement méditerranéen qu'on a pu qualifier « d'agrotown " ou " agro-ville » (PITT-RiverS, 1963).

(4) C'est par le terme " colline " que sont désignés en Basse-Provence les espaces non cultivés plus ou moins boisés, vallonnés ou non (BROMBERGER, DUFOUR et al., 1981). 
d'un point de vue géographique d'ailleurs, des communes qui se différencient peu et apparaissent comme des variantes sur un même thème ${ }^{(5)}$. Ce type de configuration spatiale traduit une structure que d'autres indicateurs confirmeront, celle d'une focalisation sur le bourg central qui possède l'ensemble des services (ou presque) nécessaires aux habitants dans leur vie quotidienne : commerces, bureau de poste, école, café(s), etc. Cette présence dans chaque commune est déjà un indicateur précieux sur la circulation des habitants, du moins sur un plan quotidien et qui a pu être confirmé par l'enquête : le dépassement des limites communales ne fait pas nécessité et relève au contraire d'un acte sortant du quotidien.

La situation géographique du canton de Comps est assez différente. Ici les communes sont composées de territoires, parfois assez vastes, où les habitations sont dispersées en hameaux de taille variée ou en grandes fermes isolées. Situés dans la zone de transition entre la Basse et la Haute-Provence, au nord du Grand Plan de Canjuers ${ }^{(6)}$, il s'agit des premiers contreforts des PréAlpes du sud. Si certains hameaux sont plus importants que d'autres et possédaient jusque dans les années 1960 une école ou une épicerie, seuls les villages de Comps et de Trigance regroupaient un ensemble de services comparables aux agro-villes du canton de Cotignac. Cette situation géographique, caractérisée par un habitat dispersé en micro-unités, forme un premier indicateur d'une nécessité de circulation interne plus grande pour les habitants que dans le canton de Cotignac. Par ailleurs, le fait que le canton de Comps se trouve au carrefour de trois départements laisse présager une plus grande conscience de certains sentiments d'appartenance " géoadministrative " que dans un canton " central " comme celui de Cotignac. L'enquête de terrain n'a pas seulement confirmé la circulation infra-cantonale, mais a également révélé une circulation des habitants vers des régions voisines et notamment vers le «bas-pays » varois ou encore vers la petite ville de Castellane, située au nord du canton dans le département des Alpes-deHaute-Provence (anciennement Basses-Alpes).

Cette circulation des habitants a formé un deuxième indicateur précieux, analysé à travers différentes sources comme la coopération agricole, les réseaux commerciaux ou encore les aires de relations matrimoniales. Ces dernières constituent en effet, dans des sociétés comme les nôtres, des indicateurs quantifiables de l'évolution historique des relations sociales, aussi bien à des niveaux infra-communaux qu'inter-communaux et (micro-) régionaux. L'analyse des relations matrimoniales dans les deux zones étudiées a ainsi permis d'affiner les indicateurs révélés par l'analyse de la situation géographique. Leur étude diachronique (depuis le milieu du XIX“s. jusquà nos jours) a mis en évidence pour les deux cantons une érosion de l'endogamie villageoise, notamment pour les périodes les plus récentes. Mais cette analyse révèle surtout que pour une même période historique les taux d'endogamie

(5) SCHIPPERS, 1986, p. 20.

(6) Qui est devenu depuis 1968-1973 une zone militaire interdite. 
villageoise dans le canton de Cotignac avoisinent les taux d'endogamie cantonale dans le canton de Comps ${ }^{(7)}$. Par ailleurs, si l'exogamie communale dans le canton de Cotignac ne semble pas désigner une micro-région bien délimitée, l'exogamie cantonale du canton de Comps fait apparaître une micro-région péri-cantonale à l'intérieur de laquelle, jusqu'aux années 1950 , près d'un(e) habitant(e) sur deux du canton a trouvé son conjoint. Les relations matrimoniales nous montrent ainsi comment du point de vue de la circulation des conjoints chacune des agro-villes du canton de Cotignac a fonctionné de la même façon que la zone cantonale et péri-cantonale de Comps ${ }^{(8)}$. L'enquête orale au sujet de l'interconnaissance des habitants des deux cantons fait écho à ces différences : dans le canton de Comps, les habitants savent situer chacun dans sa parenté de manière précise et on y affirme volontiers que "dans le canton, on se connaît tous, on est tous un peu parents "; dans les villages du canton de Cotignac cette interconnaissance, précise à l'intérieur des limites communales, devient rapidement plus imprécise au-delà.

Ces premiers indicateurs, confirmés par l'enquête orale, sont plutôt des constats (statiques) de modes de vie et en particulier de modes de circulation différents entre les deux cantons, qui nous ont incités à rechercher d'autres indicateurs (dynamiques) en amont. L'économie, essentiellement rurale dans les deux cantons jusqu'à une date récente, nous a offert quelques nouveaux indices. Dans les gros villages du canton de Cotignac, l'économie agricole est fondée sur la viticulture, complétée dans des proportions variables par l'oléiculture. A l'exception des vendanges ${ }^{(9)}$, tous les travaux agricoles y sont exécutés par le groupe familial avec l'aide éventuelle d'une main-d'œuvre occasionnelle recrutée dans la commune. Depuis le début du siècle, la transformation de la production (vinification et fabrication d'huile d'olive) y est confiée à des coopératives ${ }^{(10)}$ regroupant quasi exclusivement les agriculteurs de la commune. Ces coopératives s'inscrivent dans une tradition d'institutions communales comme les Cercles ou encore les Caisses d'Assistance Mutuelle fondées au XIX' siècle $^{(11)}$ où l'appartenance à la commune était un des critères importants d'admission, et l'égalité des membres un des principaux traits de fonctionnement. Les coopératives prennent en charge l'achat et la vente des produits agricoles, tandis que des marchés hebdomadaires dans chaque village permettent aux habitants l'acquisition de produits à usage domestique. Ici encore ces indicateurs économiques nous renvoient à un enfermement dans l'espace communal de la plupart des habitants.

(7) Où l'endogamie villageoise a été pour toute la période étudiée assez faible : 19\% entre 1890 et $1909,18 \%$ entre 1920 et $1939,19 \%$ entre 1950 et 1969.

(8) Pour l'analyse chiffrée des relations matrimoniales : cf. DUFOUR et SCHII'PERS, 1987.

(9) Qui font appel à une main-d'œuvre essentiellement étrangère, autrefois à la commune, aujourd'hui à la région, voire la nation.

(10) A Cotignac fut fondée en 1905 la deuxième cave coopérative (communale) de France, au moment de la grande crise vinicole.

(11) Cf. Durour, 1991. 
Dans le canton de Comps, la situation économique est assez différente. L'économie rurale y est fondée sur l'agropastoralisme combinant un élevage plus ou moins extensif d'ovins, un élevage laitier modeste, la culture de céréales, de fourrage, et depuis la fin du XIX' siècle de pommes de terre. La relative pauvreté de la région a contraint depuis des siècles une partie de la population à un exil saisonnier vers le bas pays ${ }^{(12)}$, exil devenu pour beaucoup définitif dans la deuxième partie du XIX' siècle. Mais jusque dans les années 1950, des agents recruteurs de grands domaines viticoles du bas pays venaient embaucher les jeunes gens du canton pour la période des vendanges, ce qui a évidemment favorisé l'interconnaissance cantonale évoquée plus haut. L'agriculture locale et surtout l'élevage ont également toujours nécessité la circulation des hommes pour acheter et vendre des bêtes dans des foires plus ou moins proches ${ }^{(13)}$. La complémentarité des productions agricoles avec le bas pays varois voisin a donné aussi à la plupart des familles du canton l'habitude de s'y rendre pour vendre fromages, pommes de terre, foin, et $\mathrm{y}$ acheter huile et vin. Lorsque au début du siècle la modernisation technique ${ }^{(14)}$ exige des regroupements d'intérêts, ils prendront la forme de syndicats d'achat et de battage réunissant des agriculteurs (aisés) des différentes communes du canton et même au-delà, selon des critères rationnels et non pas d'appartenance communale comme dans le canton de Cotignac. Plus récemment, ce furent les mêmes arguments qui ont permis la création d'une coopérative de commercialisation de pommes de terre regroupant sous un emblème commun des agriculteurs des trois cantons de Comps, Castellane et St-Auban, appartenant aux trois départements du Var, des Alpes-de-Haute-Provence et des Alpes-Maritimes.

Comme nous l'avons déjà souligné, la série des indicateurs n'est limitée que par la problématique de l'observateur et nous avons pu ainsi multiplier leur nombre dans cette étude ${ }^{(15)}$. La comparaison des deux terrains d'enquête a permis de constater que chacun d'eux avait un certain nombre d'indicateurs d'appartenance et donc de marqueurs d'identité potentiels qui lui étaient propres et semblaient n'avoir aucune valeur heuristique sur l'autre terrain. Parce que les sentiments d'appartenance et d'une identité originale impliquent toujours une situation réelle ou imaginée de mise en relation avec une différence, nous avons surtout recherché la présence ou l'absence, pour les habitants des villages étudiés, de telles situations qui permettent, justement, soit la prise en considération autochtone de certains indicateurs comme marqueurs, soit, en l'absence d'indicateurs, la création de stéréotypes. Les indicateurs d'appartenance peuvent ainsi tirer leur pertinence autant de lcur prćscncc que de leur absence, étant subordonnés à des phéno-

(12) Cf. BLANCHARD, 1945, p. 469.

(13) Principalement à Castellane et à Aups.

(14) En l'occurence l'apparition des grosses batteuses à poste fixe.

(15) Nous avons également analysé les critères de recrutement de la vie associative, la scolarisation, les connaisances anthroponymiques et toponymiques, les habitudes alimentaires et vestimentaires, voire même les attitudes corporelles. 
mènes plus généraux - qu'on pourrait éventuellement appeler des méta-indicateurs - comme dans notre étude, par exemple, la probabilité de rencontre réelle ou imaginaire avec d'Autres. Par ailleurs, les indicateurs prennent également une part importante de leur valeur dans leur articulation avec les marqueurs et les stéréotypes puisqu'il apparaît que, du moins sur les terrains étudiés ici, il existe, comme nous allons le voir, des phénomènes de compensation à la fois quantitative et qualitative entre ces trois séries d'indices.

\section{L'INVENTION DE L'AUTRE}

Traits subjectifs de différenciation, les marqueurs et les stéréotypes forment une série limitée, un système clos, représentant parmi toute une série de possibles les traits considérés par chaque groupe de référence comme significatifs d'un écart entre Lui et les Autres. On comprend aisément que, d'un canton à l'autre, ces critères "sur mesure " d'évaluation de l'identité et de l'altérité ne puissent se superposer. Nous nous bornerons donc ici à en évoquer quelques exemples, choisis dans chacun, pour illustrer la façon dont jouent différentiellement ces indices en fonction du substrat identitaire - mis en évidence par l'étude des indicateurs - existant ici et là.

\section{Au sein des cantons}

Dans le canton de Cotignac où, comme le laisse pressentir l'examen des indicateurs, on est en face d'Autres très semblables, les marqueurs d'identité et d'altérité sont rares aussi bien à l'échelle du canton que des communes qui le composent. Par ailleurs, tout désigne la commune comme unité de référence et d'affiliation majeure. Sur le plan matrimonial, c'est par l'expression d'une endogamie communale idéalisée par des expressions et des proverbes régulièrement évoqués : "Avant on se mariait entre nous "; " Marie-toi dans ton village, dans ta rue, dans ta maison si tu peux $"(16)$. Sur le plan linguistique, ce sont les mots du vocabulaire de l'appartenance, tels que " chez nous " ou " ici ", qui renvoient également à la commune. Dans la bouche d'un habitant du canton de Cotignac, le mot " pays " est toujours synonyme de village ou de commune. Parallèlement, lorsque quelqu'un quitte sa commune d'origine, on dit volontiers de lui qu'il s'est " expatrié ». Si partir du village c'est quitter sa "patrie ", c'est naturellement aussi à cette patrie communale que s'applique le mot « chauvin ». A propos du football par exemple, on invoquera le "chauvinisme de village " pour rendre compte des élans "patriotiques " des supporters des équipes locales ; plus largement, pour exprimer son attachement au " pays", on se dira " chauvin de tout ce qui est nous ". Dans le registre opposé, le vocabulaire de la différence renvoie aux mêmes limites spatiales. Ainsi, l'Autre, "l'étranger ", c'est en premier lieu l'étranger au village même sil vient du village voisin. Pourtant, si les habi-

(16) Proverbe au reste très répandu dans d'autres régions françaises. 
Les fêtes locales ont entre autres fonctions celles de renouvellement périodique d'une image de Soi que s'offre la communauté villageoise et de mise à jour des relations entre des personnes el des groupes que les habitudes quotidiennes séparent. lci, au Val lcommune périphérique du canton de Cotignac), lors de la fête de la saucisse, le pre mier dimanche de seprembre. Cliché T.K. Schippers. tants des autres villages sont bien désignés comme Autres, peu de choses dans leur comportement, leur allure, leurs modes de vie, leurs façons de parler ou de se nourrir apparaissent, aux yeux des intéressés, comme différenciatrices : " Rien ne ressemble plus à quelqu'un d'Entrecasteaux que quelqu'un de Montfort ou de Correns, ou de... " La différence entre les villages voisins, qui ne se traduit pas en marqueurs caractérisés, s'exprime en revanche dans une série impressionnante de stéréotypes, de réputations proverbiales que s'attribuent les uns aux autres les habitants des différentes communes. Au premier rang sont les blasons villageois, les sobriquets collectifs transmis par la tradition orale et dont la mémoire est particulièrement vive. Cette façon de représenter l'altérité en la figeant dans un blason caricatural, satirique ou dépréciatif, fait partie des modes d'affirmation des différences les plus universellement répandus qui fonctionnent entre individus, groupes locaux ou nations ${ }^{(17)}$.

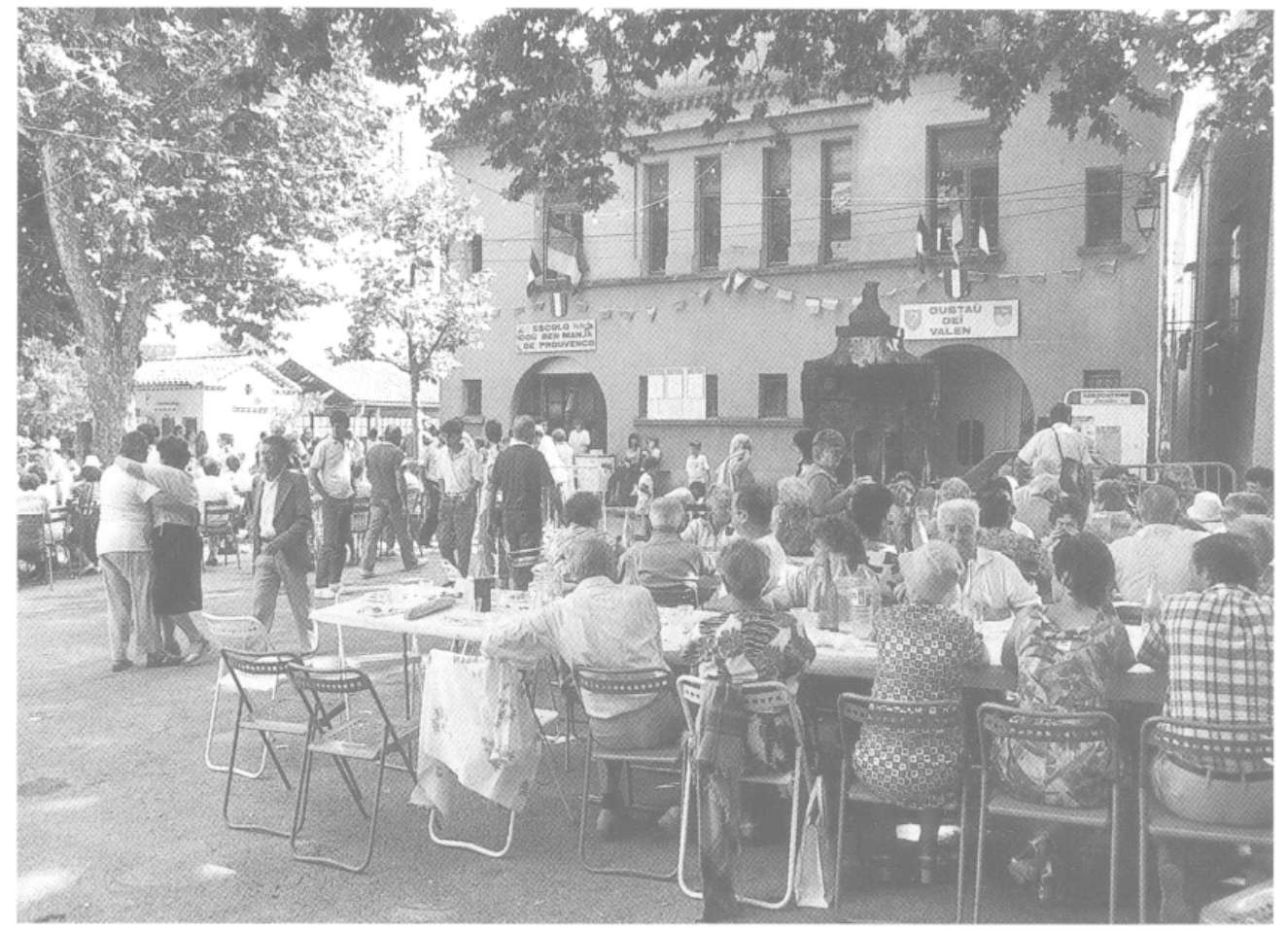

Mais au-delà de ce schéma général, comment comprendre ces stéréotypes et surtout leur profusion ? Mise à part l'image quelque peu fantasmagorique qu'ils projettent sur la réalité plus nivelée des populations de la micro-région, que nous disent-ils? On aurait tort aujourd'hui, comme on s'y essaie parfois, de traduire les blasons ou de s'attacher au contenu manifeste des réputations villageoises. La diversité des interprétations qu'en donnent les usagers est d'ailleurs révélatrice de l'importance très relative du contenu des blasons.

(17) Sur les fonctions démarcatrices des réputations collectives voir LÉVI-STRAUSS (1983), des sobriquets individucls ou collectifs voir BROMBERGER (1982), FABRE (1982), FABRE et LACROIX (1975) ; sur les représentations de l'altérité dans d'autres sociétés voir notamment Bromberger (1988) pour l'Iran, Centlivrlis (1980) pour l'Afghanistan, sur les stéréotypes nationaux Dundis (1975). 
Nul, au reste, parmi eux ne croit à la vérité des stéréotypes qu’il emploie. Contrairement aux marqueurs emblématiques qui engagent l'adhésion de leurs utilisateurs - "Ici on a la plus belle fête " par exemple -, les stéréotypes supposent une certaine distance vis-à-vis de ses affirmations que l'humour et l'ironie ne manquent pas de signaler. Il semble que ce ne soit pas - ou plus? - le contenu du stéréotype qui importe mais l'usage codé qu'on en fait. En fin de compte, l'utilisation des blasons et des stéréotypes situe les villages les uns par rapport aux autres à la fois dans leur originalité, leur individualité et leurs relations. D'une part une langue de connivence - les blasons et leurs avatars - circule entre voisins qui, à la manière des sobriquets individuels, requalifie, pour les initiés, l'ensemble de l'environnement humain proche en le rebaptisant : le plaisir évident à citer les sobriquets, à railler les défauts présumés des voisins, à évoquer allusivement tel stéréotype dépréciatif fait, à ce titre, partie d'une sorte de jeu d'humour identitaire, d'abord parce qu'il ne peut s'exercer qu'entre autochtones et donc manifester l'appartenance aux lieux, ensuite parce qu'il rappelle à toute occasion la distance entre Soi et l'Autre que l'expérience quotidienne pourrait oblitérer. D'autre part, la série des blasons et des réputations proverbiales déployée dans le canton fait apparaître des relations de proximité et d'antagonisme entre des couples de villages apparemment semblables : Correns / Montfort, Carcès / Cotignac, couples de semblables rivaux dont plusieurs indicateurs nous ont appris qu'ils étaient les plus fortement liés à l'intérieur du canton. Là encore se manifeste un processus bien connu de l'affirmation des différences qui consiste à se distancier d'autant plus de l'Autre qu'il est semblable et proche ${ }^{(18)}$.

La profusion de blasons et de stéréotypes, la vigueur de leur utilisation renvoient au même type de phénomène. Non seulement l'analyse des indicateurs ne fait pas apparaître de fortes spécificités des modes de vie, des moyens d'existence, des paysages etc... entre ces différentes communes, mais encore leurs habitants ne reconnaissent pas de différences essentielles, consciemment affirmées (marqueurs), entre eux-mêmes et leurs voisins. Rien ne ressemble plus, on l'a vu, à quelqu'un d'Entrecasteaux que quelqu'un de Montfort ou de Correns...Rien non plus ne manifeste à leurs yeux, dans leur propre village ou chez ses habitants, une singularité telle qu'elle puisse être érigée en symbole d'une identité reconnue par tous. Pourtant cette absence de traits objectifs de différenciation, cette pauvreté des marqueurs d'identité et d'altérité n'empêchent pas l'existence d'une conscience très forte d'appartenir à son village, premier pôle d'identification. Quel moyen reste-t-il alors d'exprimer une identité si fortement ressentie ? D'une part, l'évidence qui n'appelle pas de démonstration du type : "Ici c'est mieux quailleurs "; d'autre part, l'affirmation différentielle de son identité en créant symboliquement un Autre puisqu'il n'existe pas ou du moins que l'expérience quotidienne permet mal de saisir son altérité. Lutilisation des blasons, des proverbes sur les voisins participe de cette production symbolique de l'Autre, utilisation d'autant plus grande qu'est grand ici le danger de la confusion.
Il arrive souvent que le blason dép-éciatif venu de l'extérieur soit réapproprié comme rarqueur emblématique. Ainsi Correns qui a fait de l'étiquette imposée - les Pesco luno - sa propre étiquette en nommant de cette façon l'un des vins de son terroir.

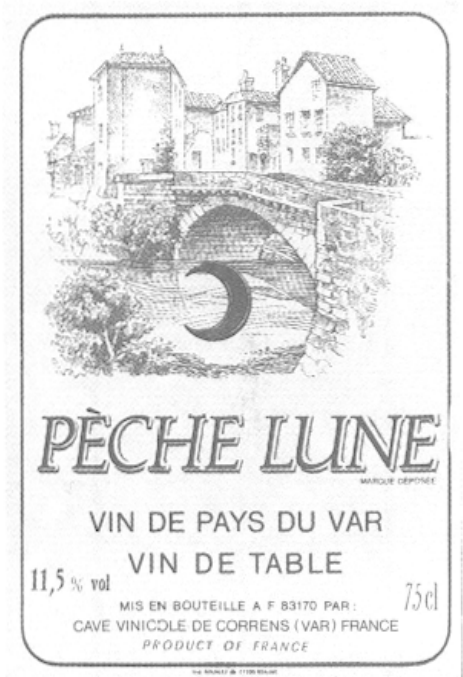


Les chorales, les fanfares, les cliques, comme les sociétés de musique de la fin du siècle dernier, sont des supports et des symboles de l'existence collective des vitlages. Ici, la Clique des Pompiers du Val défilant pour la foire de début septembre. Cliché T.K. Schippers.

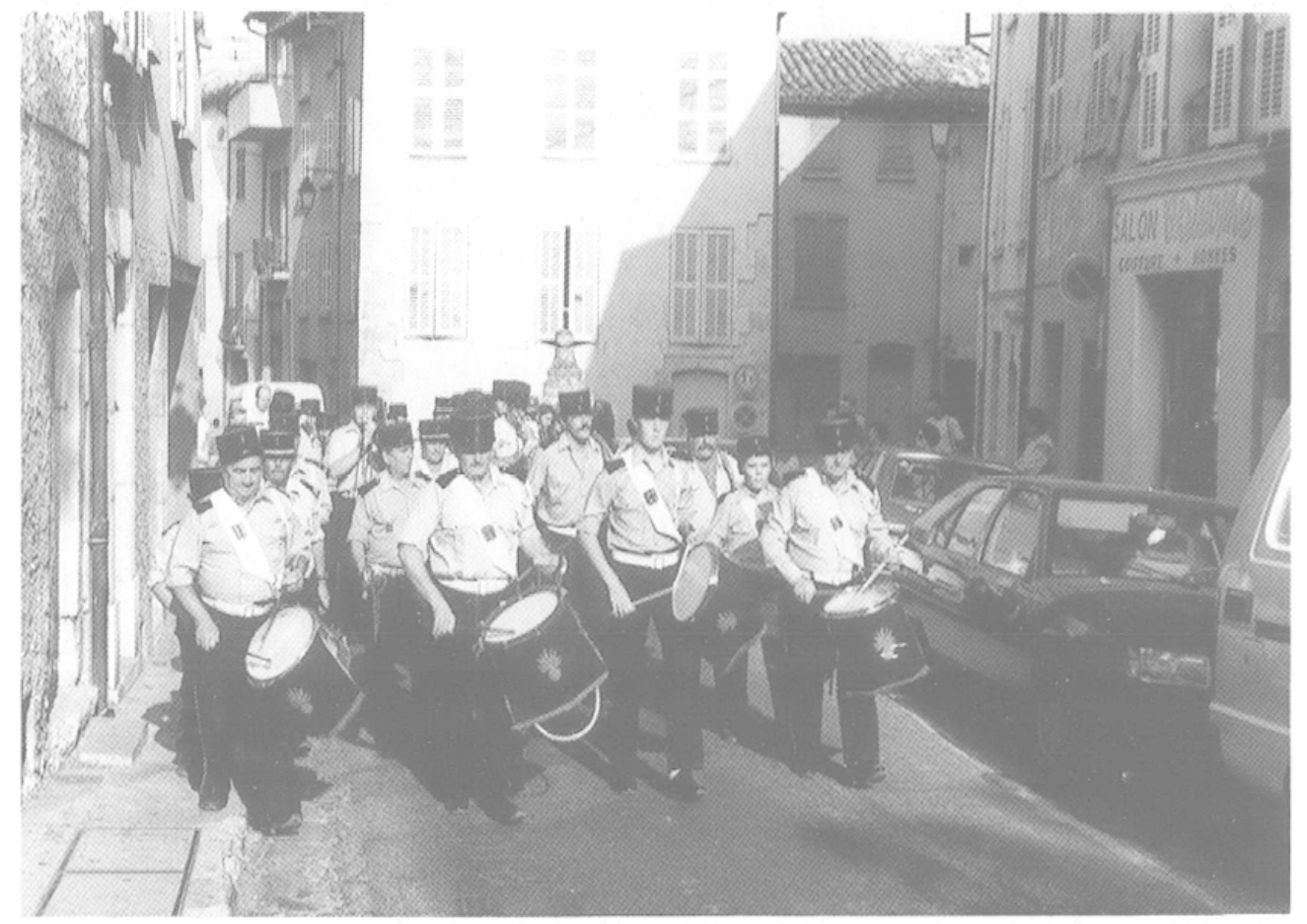

Dans le canton de Comps, en revanche, la différence décelée au niveau des indicateurs se confirme dans l'utilisation des marqueurs et des stéréotypes. Nombreux sont en effet les critères "objectifs " repris par les habitants du canton pour se différencier d'une part du reste du Var, d'autre part des deux cantons voisins.

Depuis sa création au lendemain de la Révolution, l'ensemble cantonal semble l'unité de référence utilisée à la fois par l'administration centrale et par les habitants du canton. Ceci n'exclut évidemment pas des différenciations infra-cantonales plus ou moins marquées, mais indique déjà une différence très nette avec d'autres micro-régions varoises. Les premiers traits à la fois revendiqués par les habitants et attribués par les Autres sont liés à la situation géographique du canton de Comps. Celui-ci est perçu par ses habitants comme un " haut pays " d'où l'on descend aussi bien pour aller vers le sud (vers le reste du département du Var) que vers le nord (vers Castellane et le département des Basses-Alpes). Malgré un relief assez tourmenté, le canton est donc considéré comme un haut plateau, dont le Grand Plan de Canjuers forme la partie méridionale et les vallons autour de la montagne de Brouïs la partie septentrionale. De ce premier trait découle immédiatement un deuxième, surtout mis en valeur par "ceux d'en bas " (notamment du Var), et qui désigne le canton comme une région froide : "Chaque fois qu'on venait à Bargemon, ils nous demandaient : "Alors, vous avez encore de la neige là-haut ?" "Bien que les habitants du canton de Comps ne se considèrent pas comme des " montagnards", ils leur sont associés par les gens des villages méridionaux puisqu'ils viennent du nord, de la direction des Alpes. La venue saisonnière d'une partie de la population de Haute-Provence pour se louer ou mendier l'hiver en Basse-Provence a renforcé chez " ceux d'en bas " l'idée que c'est surtout le froid (et ses conséquences économiques) qui fait descendre les " montagnards" vers les régions chaudes. Le climat froid, 
synonyme de pauvreté, s'oppose ainsi à la chaleur synonyme de bien-être, de richesse.

Mais depuis la fin du XVIII siècle et durant le XIX'e, ce facteur climatique a aussi acquis deux autres connotations : celles de pureté et de santé. La logique populaire (19), qui forme les stéréotypes en associant sans complexe la santé physique à la santé morale, a ainsi créé l'image - mille fois reproduite depuis ${ }^{(20)}$ - du canton de Comps comme une région du Var pauvre mais pure, éloignée des maladies et de la corruption morale des gros bourgs du Sud. Un certain retard économique et une présence plus marquée de l'Eglise n'ont fait que renforcer ces stéréotypes. Lorsqu'on évoque le froid face à un interlocuteur venu du canton de Comps, on résume en quelque sorte un ensemble de faits et de stéréotypes qui mêlent de manière subtile admiration et moquerie.

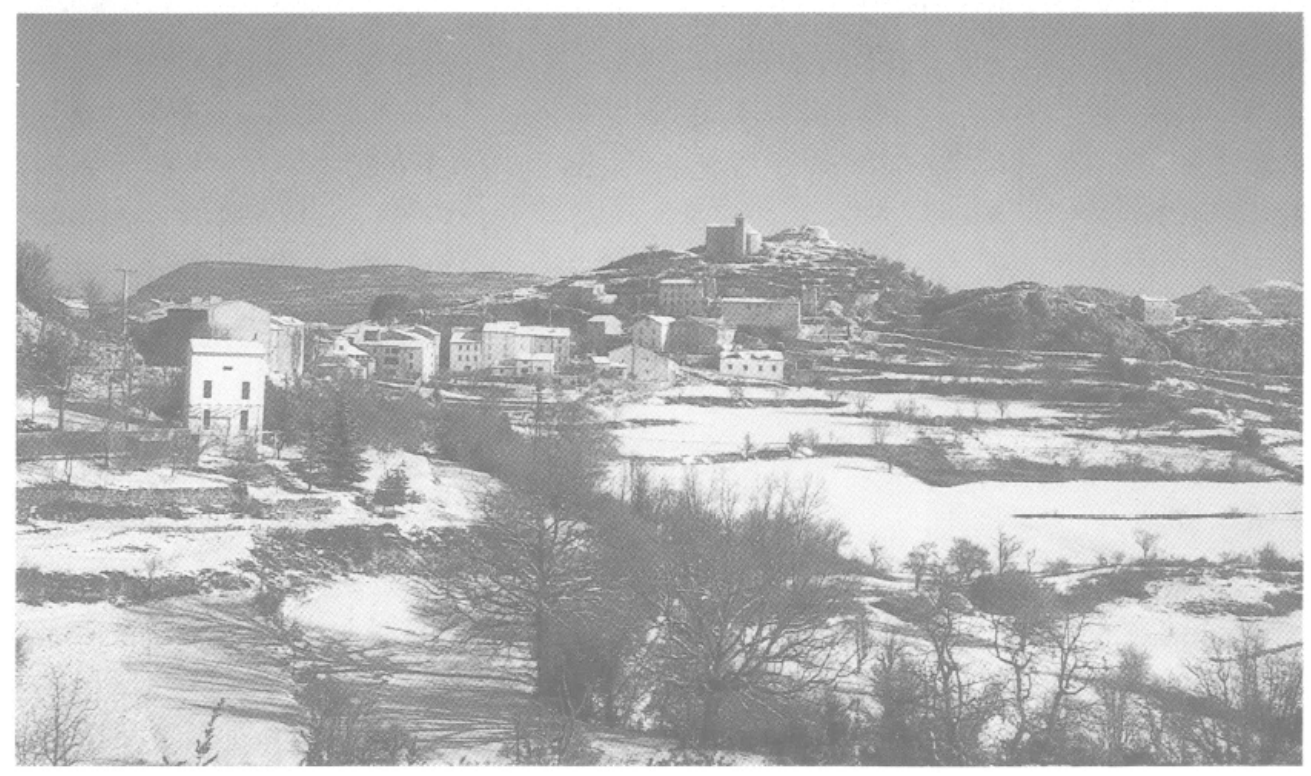

Ce mélange de sentiments est présent dans le terme par lequel de façon générale les habitants de Basse-Provence désignent ceux de Haute-Provence, le terme Gavot. Ce mot a plutôt une consonance péjorative et est ainsi habituellement employé pour désigner les Autres, ceux qui habitent plus haut. Il est alors frappant de voir que les habitants du canton de Comps, interrogés sur les différences identitaires avec le bas pays, se désignent eux-mêmes par le terme Gavouat: "On n'est quand même pas des Provençaux ici, on est des Gavouat. "Le terme Gavouat est donc connu et utilisé par les habitants du canton pour se différencier de ceux du bas pays, sans qu'il soit pour autant revendiqué : "Ici, on appelle les gens de cette région li Gavouat et en bas ce sont li Provençau. Mais ils nous apprennent rien, je vous le dit franchement, ni en nourriture, ni en rien du tout ! "Le terme Gavouat est surtout employé pour se démarquer des autres Varois désignés comme Provençaux, tandis que ceux qui habitent vers le nord sont désignés par le terme li Bas-alpin.

(19) Soutenue en cela au XIX`s. par l'idéologie dominante.

(20) Par exemple dans les romans qui se déroulent dans cette région, comme ceux de Gaston Cauvin (1945, 1954), ou encore dans les guides touristiques (HeNSELING, 1929). 
Ces sobriquets désignant les habitants du canton traduisent leur double appartenance ou plutôt ce que l'historien Judt (1978 : 14) a appelé the inbetween character du canton, qui est à cheval sur la Haute et la BasseProvence. L'auto-identification s'appuie souvent sur cette originalité d'être ni tout à fait comme les uns ni tout à fait comme les autres. L'identité locale est ainsi très fortement marquée par cet aspect bi-référentiel qui apparaît par exemple au niveau de la langue mais aussi de la cuisine, de l'habitat ou du paysage. La différenciation culinaire notamment avec le bas pays est un bel exemple de la reprise d'un indicateur comme marqueur. Ainsi la pomme de terre a acquis depuis son introduction une valeur emblématique très forte, non seulement comme produit commercialisable dans le bas pays, mais aussi comme élément souvent jugé indispensable dans la nourriture quotidienne. Les récits des émigrations saisonnières vers le bas pays évoquent souvent " qu'en bas ils font la soupe [du soir] avec des courges et de l'huile, tandis qu'ici nous mettons toujours des pommes de terre et un morceau de cochon. " Pour illustrer cette différence alimentaire avec les habitants du bas pays et leur "ignorance " en matière de pommes de terre, ces mêmes récits soulignent fréquemment une différence linguistique : "Ici on dit la trufo, en bas ils disent les patates » ${ }^{(21)}$. L'altérité culinaire ${ }^{(22)}$ est ainsi en quelque sorte renforcée par l'altérité linguistique.

A côté des aliments très marqués à cause de leur lien direct avec la production locale, il y a aussi des préparations culinaires qui témoignent de l'appartenance du canton à des aires différentes. Ici se retrouve sa double appartenance culturelle ; certains plats sont typiquement haut-provençaux tandis que d'autres sont surtout associés symboliquement à la Provence orientale. Le premier plat local cité comme tel par les informateurs est les croüsses (crouset), pâtes fabriquées avec du blé tendre et consommées avec des noix pilées lors du Gros Souper de Noël. Ce plat, uniquement attesté en Haute-Provence, a surtout une valeur emblématique vis-à-vis du bas pays varois. En revanche, la préparation du chou faģun ${ }^{(23)}$, dont la " patrie " est la ville de Grasse, rapproche le canton culinairement de la région de Grasse, et notamment du canton de Fayence. Un pain que les habitants du canton considèrent comme une marque de différence identitaire est la fougasse à l'anchois, pain plat enduit d'anchois écrasés, " qu'on ne trouve que dans les Basses-Alpes ». L'achat et la consommation de la fougasse à l'anchois, lorsqu'on va à la foire de Castellane, sont un des signes qu'on est " chez les Autres ". La consommation de plats stéréotypés appartenant à des aires de distribution géographique différentes symbolise bien ainsi the in-between character du canton.

Les études dialectologiques, en faisant converger sur les cartes les lignes isoglosses vers le canton, apportent le témoignage linguistique de la multipli-

(21) En provençal du bas pays varois on emploie le terme poumo de terro ou patato tandis que dans le bas pays niçois on dit tartiflo.

(22) Qui est évidemment aussi un indicateur " objectif ".

(23) Chou évidé, farci et cuit à l'eau, enfermé dans un filet. 


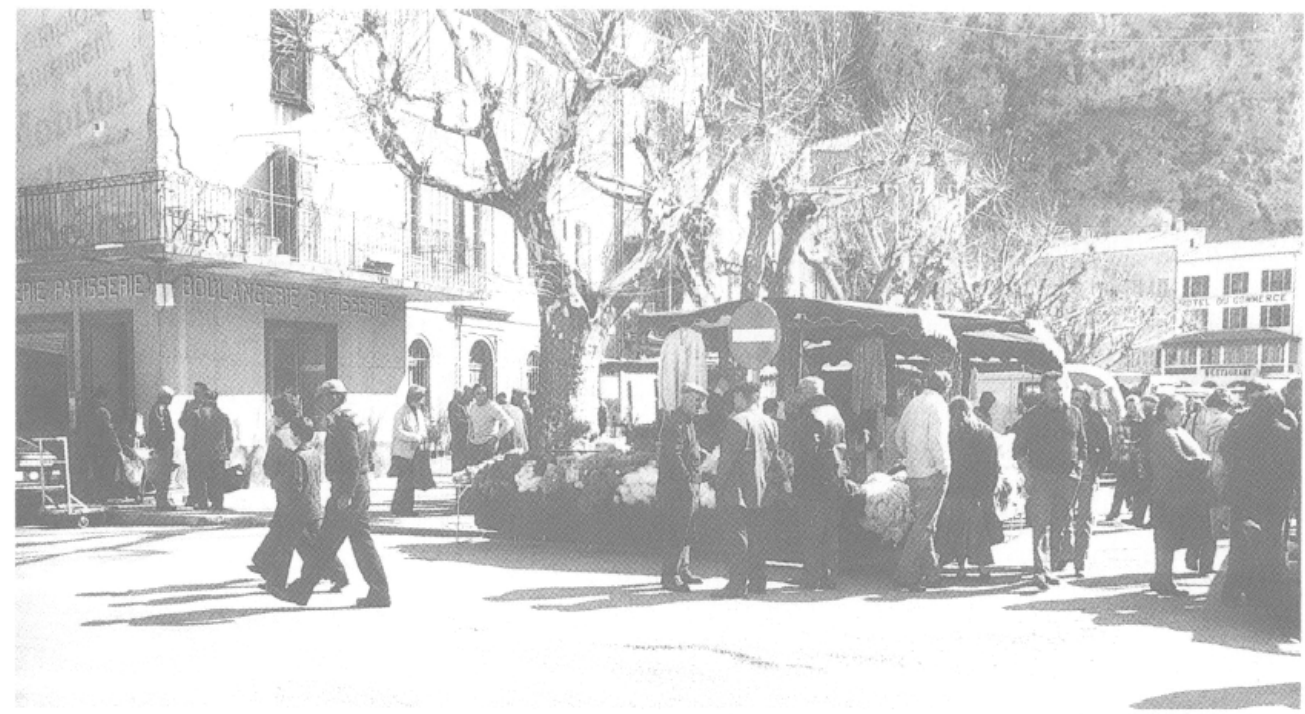

cité des influences dont il est le carrefour. Pour les habitants, des nuances à la fois phonétiques et syntaxiques leur permettent néanmoins de se reconnaitre et de distinguer le parler des régions voisines proches. Parmi les exemples de différences linguistiques reconnues par les habitants, on peut remarquer qu'il s'agit souvent de mots qui ont aussi une certaine connotation emblématique en soi comme la pomme de terre déjà citée ou bien la brebis - l'élevage ovin étant une activité importante aussi bien dans le canton que dans les BassesAlpes - qu'on appelle fedo dans le canton (et dans le reste du département du Var) et $f e$ dans les Basses-Alpcs. Globalcment, lcs habitants du canton se sentent linguistiquement plus proches du reste du Var que des Bas-alpins, réactualisant ainsi un découpage administratif antérieur à la République.

Le canton de Comps se distingue donc de plusieurs manières de l'autre canton varois étudié. Cette différence repose essentiellement sur les interrelations entretenues avec les habitants des micro-régions voisines. Dans le canton de Comps, situé géographiquement dans la zone de transition entre la Basse et la Haute-Provence, les éléments sur lesquels s'appuie l'identité locale varient souvent en fonction de l'Autre auquel on est confronté. Indicateurs, marqueurs et stéréotypes sont ainsi parfois mono-référentiels, fréquemment bi-référentiels, voire pluri-référentiels. Lidentité locale se traduit alors surtout par la maîtrise commune aux habitants de tous les villages du canton de l'ensemble des références différenciatrices. Les "Nous d'ici " ne s'opposent pas, comme dans le moyen Var du canton de Cotignac, à des Autres à la fois semblables et peu différenciés, mais au contraire ils se distinguent de chaque ensemble d'Autres de manière spécifique. Les sentiments d'appartenance et d'identité locales forment chez les habitants du canton de Comps des catégories directement interliées à la dynamique des relations (socio-économiques) avec les différents groupes d'Autres, et non pas des catégories figées dans une individualité entourée de semblables.

Une des conséquences de la situation géographique particulière du canton est en effet l'occurrence différenciée des marqueurs selon les ensembles auxquels on s'oppose. Ainsi par exemple, le découpage administratif est utilisé
Foire de Castellane où les "Varois" du canton de Comps rencontrent leurs amis "basalpins " du canton voisin let achètent des fougasses à l'anchois dans la boulangerie située à gauche de la photol.

Cliché T.K. Schippers. 
pour se différencier des habitants des deux départements voisins tandis que l'agriculture est retenue comme marqueur d'identité à l'égard du reste du Var. L'appartenance stéréotypique rattache en revanche les habitants du canton aux cantons des départements voisins dont les modes de vie sont similaires. La situation du canton au sein de la zone de transition entre la Haute et la Basse-Provence rejaillit à de multiples occasions, sous la forme de sentiments d'appartenance bi-référentiels. Cette double référence est un constituant majeur de l'identité locale. Son originalité réside dans le fait qu'elle n'est pas seulement fondée sur une série de caractères propres au canton mais sur des oppositions circonstancielles avec les régions voisines. La variété des vecteurs de l'identité des habitants du canton de Comps est ainsi le reflet de celle des ensembles qui l'entourent, à l'image de ce caméléon imaginaire qui voudrait, contrairement à ses congénères réels, toujours se démarquer de l'environnement où il est amené à séjourner.

\section{Au-delà du premier cercle}

La mise en rapport des indicateurs, marqueurs et stéréotypes, telle qu'elle a été envisagée jusque-là, au niveau du plus petit cercle de référence, montre bien que les mécanismes de production de l'identité n'utilisent pas dans les mêmes proportions, selon les terrains, les séries objectives et subjectives de critères de définition de Soi et des Autres. Le poids respectif de ces trois types d'indices varie selon les caractéristiques du lieu. Mais l'on peut aller plus loin dans l'observation de ces variations : le fonctionnement et le rôle de ces indices ne jouent pas non plus de la même façon selon le niveau d'appartenance considéré. Lorsque l'on change d'échelle, les mécanismes d'identification et de différenciation jouent en effet autrement. Ce phénomène est particulièrement bien observable à Cotignac où des cercles d'appartenance emboîtés se dessinent de façon très nette. C'est donc ce canton qui nous servira d'exemple.

Pour Cotignac, passée la frontière communale, le second cercle d'appartenance est le département du Var. Cette reconnaissance du Var comme première unité d'affiliation extra-communale s'exprime très nettement dans l'identité qu'on donne de soi en réponse à une question directe mais se lit aussi, a contrario, dans les façons de considérer l'étranger. Est déjà moins étranger celui qui peut, par son accent, des références topographiques, amicales, familiales, professionnelles ou sportives ${ }^{(24)}$, sinscrire dans le vaste réseau des relations varoises et, de ce fait, fournir les repères de son appartenance. Mais c'est surtout différentiellement que ce Var se définit par rapport aux régions qui l'entourent et en premier lieu par opposition avec la « montagne " toute proche : les Alpes-de-Haute-Provence.

Cette frontière sépare les Varois d'un monde perçu comme radicalement différent du leur par la rudesse de son climar, celle de ses habitants, des paysages singuliers où se signale notamment l'absence de vignes et d'oliviers, des

(24) Suscitées par les concours de boules et les matches de football en particulier. 
modes de vie et de faire-valoir en contraste avec les leurs (éleveurs-céréaliers versus agriculteurs-viticulteurs), une façon de parler, un accent jugés très particuliers. C'est le pays "gavot " déjà évoqué. Outre ces distinctions qui, somme toute, ne font que confirmer une série de contrastes objectivement établis, le pays gavot fut longtemps, on l'a dit, le pays voisin dont les habitants émigraient périodiquement, puis définitivement, pour gagner leur vie plus bas. Si le Gavouat est étranger, c'est aussi l'étranger qui, se déplaçant, est connu sous toutes les facettes de son étrangeté. Cette fréquentation de la différence a alimenté, plus que pour d'autres régions, des attitudes collectives et des stéréotypes particuliers. Rude, peu bavard, il est qualifié de froid, plus pauvre, plus soucieux de garder un pécule qu'il est descendu gagner, il est âpre au gain, extérieur à la société où il est de passage et à son style de sociabilité, il est sauvage. Cela, doublé de sa proximité géographique, fait que, selon le principe déjà observé à l'échelle des communes, c'est de lui qu'on se distingue le plus vigoureusement.

A l'opposé, au sud, ce sont les Marseillais qui forment le second pôle d'altérité dont on se démarque nettement ; Marseille et non les Bouches-du-Rhône ni une aire plus vaste. La langue, l'accent en particulier, constituent le principal trait d'opposition perçu comme tel. C'est ensuite sur le tempérament des gens (hâbleurs, envahissants), le caractère ostentatoire des comportements - les Marseillais se "croient " plus que les autres -, l'urbanité et ses connotations habituelles que se fonde le sentiment d'altérité. Marseille, par ailleurs, représente la ville d'émigration la plus importante après Toulon depuis l'exode rural. Comme le Gavot mais pour des raisons inverses, le Marseillais est donc "l'étranger " le mieux connu et ce d'autant plus aujourd'hui que, comme d'autres citadins, il migre lui aussi régulièrement pour ses loisirs, et notamment la chasse, vers ces régions de l'intérieur ${ }^{(25)}$.

A ces oppositions très fortes avec la montagne du nord et la métropole régionale du sud s'ajoutent celles, plus nuancées, avec les $N$ içard ${ }^{(26)}$ à l'est et la Provence rhodanienne identifiée comme la Provence de Frédéric Mistral à l'ouest. Aux Niçard on reconnaît un parler tellement différent de celui existant dans le Var qu'il en devient parfois incompréhensible. Cette différence, on l'attribue surtout à l'influence de l'Italie proche ${ }^{(27)}$, laquelle d'ailleurs n'affecte pas seulement la langue mais aussi la cuisine, voire une façon d'être, elle-même pensée comme mâtinée de traits transalpins. De la Provence rhodanienne, à laquelle on s'oppose surtout sur des critères linguistiques, on retient surtout Arles et Avignon, parfois Maillane, berceau de Frédéric Mistral et du mouvement félibréen.

(25) Aussi, pour situer son origine à l'extérieur de chez soi fait-on référence à Toulon en se démarquant vigoureusement de Marseille.

(26) Nom des habitants de la ville de Nice et par extension du Comté de Nice en langue vernaculaire.

(27) Linguistiquement, le parler du Comté de Nice se rattache au dialecte provençal, point de rencontre du provençal méditerranéen et du provençal alpin. 
Mais qu'il s'agisse de Nice ou d'Arles, les distinctions perçues contribuent à définir des limites au-delà desquelles on sent nettement que "ça n'est plus pareil ", sans que ces différences n'éveillent antagonisme ou dérision. Il semble plutôt que ces régions, avec lesquelles on n'a d'autres contacts qu'occasionnels (voyages, service militaire...) et une connaissance le plus souvent indirecte, constituent seulement des frontières sans potentialités négatives qui par elles-mêmes sont suffisamment lointaines pour qu'on n'éprouve pas le besoin de s'en démarquer. A ce niveau d'appartenance extracantonale, les mécanismes de différenciation jouent donc de façon plus évidente qu'à un échelon plus réduit :

- Alors que les stéréotypes et les réputations proverbiales inspirent une certaine réserve à l'échelle des rapports inter-communaux, on observe une relative concordance entre la perception des usagers et les stéréotypes habituellement appliqués aux populations dont ils se différencient : le Gavot pauvre, froid, sauvage et travailleur, le Marseillais hâbleur et envahissant, le Niçois mâtiné d'Italien... tout cela correspond bien à des images stéréotypées largement répandues dans toute la Provence.

- Par ailleurs, alors que la conscience de soi s'appuie fort peu sur des indicateurs aréologiques à l'échelle du canton, il y a globalement coïncidence entre les distinctions perçues par les usagers et les ensembles objectivement existants à l'échelle de la Provence. A ceci près que cette géographie de la différence semble dessiner des cartes incomplètes où émergent des points clés fortement individualisants (par contraste) et qui laissent dans l'ombre, c'està-dire dans l'indifférence, des zones entières desquelles le sentiment d'appartenance n'a que faire pour s'alimenter. Les références à Marseille, Nice, au pays gavot, Arles, Avignon ou Maillane dénotent bien la perception d'ensembles contrastés, mais en condensant sur ces îlots les représentations autochtones qu'une cartographie culturelle " objective " étalerait davantage en Provence littorale, maritime, alpine ou rhodanienne par exemple.

- Enfin certains traits culturels, comme la langue dont on a vu qu'elle ne constituait pas un marqueur d'identité à l'échelon communal ${ }^{(28)}$, deviennent, à un niveau supérieur d'insertion, un signe de démarcation ou de reconnaissance. Signe de démarcation lorsque l'on établit sur des critères linguistiques la distance entre soi et les régions voisines : “Nice, Marseille, c'est carrément différent "; signe de reconnaissance lorsque par delà ces différences c'est l'usage de la langue qui est envisagé : " même étant différent, on parle patois quand même ».

La dialectique du Nous et des Autres se manifeste par conséquent de manière distincte selon les ensembles auxquels elle s'applique. Au niveau minimal, celui des relations inter-villageoises où les contrastes objectifs sont faibles (peu d'indicateurs pertinents), les traits discriminants sont fins et difficilement extériorisables (absence de marqueurs emblématiques " actifs"), si

(28) Y compris lorsque des différences objectives de vocabulaire ou de prononciation existaient entre les communes. 
ce n'est par des expressions tangentielles (marqueurs emblématiques "passifs " ou "attributs" emblématiques). A ce niveau de proximité avec l'Autre, la distance nécessaire à la constitution d'une identité différentielle est créée par la production d'une altérité symbolique (abondance des stéréotypes). Au niveau supérieur, celui des relations intra-régionales, les traits discriminants apparaissent plus nettement, sont mieux ressentis (marqueurs linguistiques, économiques...) et la constitution d'une identité, varoise dans ce cas, s'appuie sur la perception d'une altérité vécue à la fois sur deux registres : celui des différences culturelles " objectivement " fondées et celui des représentations stéréotypiques et des jugements de valeurs déduits de ces différences.

Mais si ces deux registres coexistent aux deux niveaux considérés, ils ne participent pas dans les mêmes proportions à la constitution de l'image de l'Autre. Au premier niveau où la connaissance empirique de l'Autre, nourrie par les contacts quotidiens et un voisinage de longue durée, interfere avec l'altérité qu'on lui prête, l'ordre des représentations domine et il existe un hiatus entre l'Autre vécu et l'Autre représenté : les stéréotypes, les réputations proverbiales qui marquent l'altérité inter-villageoise ne supposent pas, comme on l'a vu, la croyance en leur vérité. Au niveau intra-régional, au contraire, les deux registres où puisent les mécanismes d'identification de l'Autre sont équivalents et les stéréotypes sont " vrais " parce qu'ils découlent de l'interprétation de différences "objectivement " constatées. Que cette interprétation ne coïncide pas obligatoirement avec la réalité n'a pas beaucoup d'importance, les lointains où se situent ces Autres ne remettant pas plus en question la " véracité " des stéréotypes que celle des différences culturelles. Mieux encore, quand ces lointains se " rapprochent ", par l'effet des migrations par exemple comme dans le cas des Gavots ou des Marseillais, les stéréotypes sont aussi vivaces qu'est vive la volonté de démarcation et il semble que, dans ce cas, la fréquentation de l'Autre conforte et renforce son altérité. C'est aussi ce que l'on a pu constater à un niveau plus global dans l'expression des stéréotypes nationaux que n'entament pas l'expérience concrète des habitants des pays concernés (Dundes : 1975).

Si l'on tente d'esquisser un premier bilan à la suite de cette étude, quelles remarques peut-on formulcr? La mise en perspective de deux terrains a conduit à évaluer les différents degrés de pertinence des indicateurs et des marqueurs selon les lieux. Bien qu'appartenant à un même ensemble, les indices marquant l'identité locale s'y manifestent différemment. L'analyse comparative des indicateurs, des marqueurs et des stéréotypes montre donc l'élasticité des références identitaires. Elasticité territoriale d'abord puisque dans un cas cette notion recouvre la commune, dans l'autre une unité plus large ; élasticité perceptuelle ensuite puisque à l'intérieur de deux ensembles administratifs identiques les références se structurent différemment : dans le cas 
du canton de Cotignac, selon un modèle rayonnant à partir de pôles similaires juxtaposés et relativement autonomes ; dans le cas du canton de Comps il s'agit plutôt d'un réseau à ancrages multiples - villages et hameaux polarisé autour d'un centre, le chef-lieu de canton. Dans la première situation, des indices d'identité émis à partir de pôles centraux - les communes s'atténuent du proche au lointain jusqu'à disparaitre au niveau cantonal et au-delà. Lidentité locale ne s'y exprime que dans la dialectique entre communes voisines sans former d'unités de référence à un niveau plus englobant. Dans le canton de Comps, les indices d'identité semblent plutôt s'appuyer sur des réseaux, comprenant tous les habitants du canton, polarisés autour du chef-lieu qui rassemble sous sa bannière l'identité commune. Le canton forme donc ici un "pays " nommé devant lequel s'effacent, du moins à l'extérieur, les individualités communales. L'existence de ce " pays » ne procède pas pour autant de la négation des hameaux et communes mais s'appuie sur eux. L'histoire récente du canton, notamment l'implantation du camp militaire de Canjuers, bien qu'enlevant au chef-lieu sa position centrale, n'a pas modifié cette identité " réticulaire " mais l'a simplement déplacée autour d'un nouveau pôle incarné par le carrefour de trois cantons voisins.

Au-delà de l'étude de ces deux cantons varois, cette recherche aura permis l'amorce d'une réflexion méthodologique plus générale sur les manières d'aborder les phénomènes d'identité dans une perspective proprement ethnologique. Lapproche tridimensionnelle de l'identité d'un groupe permet une différenciation des critères pris en compte selon une dialectique entre des traits différenciateurs " objectifs » et des traits " perçus et/ou retenus " par la population étudiée.

La valeur heuristique de cette approche ne peut se concevoir que si l'on prend en compre en même temps ces trois types d'indices et qu'on les analyse dans leur articulation réciproque. En effet, la prise en compte de ces éléments isolément, non seulement ne permettrait pas d'aborder l'identité et l'appartenance locales dans la pluralité de leurs dimensions, mais encore laisserait échapper la relation dynamique existant entre ces trois indices dans le processus d'élaboration des images de Soi et de l'Autre. Les exemples étudiés suggèrent ainsi une hypothèse qui mériterait d'être mise à l'épreuve d'autres terrains et d'autres situations, selon laquelle les sentiments d'identité s'appuient sur des traits identifiables de l'extérieur (indicateurs et marqueurs) et sur des traits issus de l'imaginaire collectif intérieur (stéréotypes), mais dans des proportions variables selon les caractéristiques intrinsèques des lieux et des situations. En d'autres termes, si les sentiments d'appartenance et la désignation de Sui et des Autres correspondent bien à une tendance générale des groupes sociaux les plus variés, la manière dont ils s'élaborent dépendraient étroitement des données sur lesquelles ils peuvent s'appuyer localement. Là où les traits objectifs de différenciation sont nombreux et les traits subjectifs forts, l'invention de l'Autre (stéréotypes) n'a pas ou peu besoin d'exister. A l'inverse, les traits sur lesquels la conscience de soi prend appui seront d'autant plus le fruit de l'imaginaire (stéréotypes) que les traits objectifs ou reconnus de la différenciation font défaut. Se définir et désigner les 
Autres ressemble ainsi à un jeu dont les antagonistes renouvellent ou réinventent continuellement les pièces pour en assurer la perpétuation.

\author{
Annie-Hélène Dufour et Thomas K. SCHIPPERS \\ URA 1346 du C.N.R.S. \\ Université de Provence
}

\title{
BIBLIOGRAPHIE DES OUVRAGES CITÉS
}

BLANCHARD (R.),

1945 Les Alpes occidentales, t. IV : Les Préalpes du Sud, Paris.

BROMBERGier (C.),

1982 "Pour une analyse anthropologique des noms de personnes", Langages, 66, pp. 103-124.

1985 "Identité alimentaire et altérité culturelle dans le nord de l'Iran. Le froid, le chaud, le sexe et le reste", Identité alimentaire et altérité culturelle, Recherches et travaux de l'Institut d'Ethnologic, n ${ }^{\circ}$ 6, pp. 5-34.

1988 "Comment peut-on ctre Rasti ? Contenus, perceptions et implications du fait ethnique dans le Nord de l'Iran ", Le fait ethnique en Iran et en Afghanistan, Actes du Colloque, Paris, pp. 89-107.

1993 "L'ethnologie de la France et le problème de l'identitć ", Actes du Colloque de Bucarest, juillet 1991 , Civilisations, Bruxelles.

Bromberger (C.), Durour (A.-H.), et alii, 1981 "Les paysans varois et leurs collines. Les enjeux symboliques d'une "passion" ", Forêt Méditerranéenne, $\mathrm{t}$. III, $\mathrm{n}^{\circ} \mathrm{l}$.

CAuvin (R.),

1945 L'homme clair, Avignon.

1954 Le marchand d'étoiles, Avignon.

Centiavris (P.),

1980 “Identité et image de l'autre dans l'anthropologie populairc en Afghanistan ", Revue Européenne des Sciences Sociales et Cahiers Vilfredo Pareto, t. XVIII, 53, pp. 29-41.
Durour (A.-H.),

1991 "Faire cuve commune", Les Coopératives vinicoles varoises, Marseille.

Durour (A.-H.), Schippers (T.-K.), 1987 Nous ici et les autres. Formes d'identité locale et microrégionale en Provence varoise, Aix - Paris.

DUNIDES (A.),

1975 "Slurs international : folk comparisons of ethnicity and national character ", Southern Folklore Quartely, 39, pp. 15-38.

F $A$ BRE (D.), Préface à AChaRd (C.), 1982 Les uns et les autres, Dictionnaire satirique des sobriquets collectifs de l'Hérault, Béziers.

FABRF (D.), LACROIX (J.),

1975 "L'usage social des signes", Communautés du Sud, Paris, pp. 564-593.

Hinseling (L.),

1977 Zigzags dans le Var, Marseille, 1 éd. Toulon, 1929-1935.

JUDI (T.),

1978 Socialism in Provence, 1871-1914, Cambridge.

LABOV (W.), 1981 Sociolinguistique, Paris.

Le Monde alpin et rhodanien, $1988 \mathrm{La}$ moquerie. Dires et pratiques, n'3-4, Grenoble.

LÊVI-SIRAUSS (C.), 1983 Le regard éloigné, Paris.

PITT-Rivirs (J.) et collab., 1963 Mediterranean country-men, Paris La Hayc, Mouton.

SCHIPPERS (T.-K.),

1986 Temps vécus, temps perçus. Au fil des saisons en Provence intérieure, Paris. 